\title{
Development of Urban Logistics Distribution under New Retail
}

\author{
Weimin Di, Fu Li \\ Institute of Administrative Engineering College, Zhengzhou University, No.100 science Avenue, Zhengzhou, \\ China
}

Keywords: new retail, urban logistics distribution, big data, intelligent logistics

\begin{abstract}
Against the new retail production background, meaning and characteristics, the shortage of urban logistics and distribution, and the problem of urban distribution logistics restricting new retail development has been found. Combined with the research results of domestic scholars, this paper puts forward some reflections and proposals for the development of urban logistics distribution under the new retail.
\end{abstract}

\section{INTRODUCTION}

According to the data released by National Bureau of statistics, China's total retail sales of social consumer goods growth declined from $18.3 \%$ in 2010 to $10.7 \%$ in 2015, the top 100 enterprises nationwide chain retail growth from the 2013 growth rate below 10\% for the first time, an increase of only $4.3 \%$ in 2015 , the growth rate is the lowest year since 100 enterprises nationwide chain statistics. With labor costs and housing rental costs rose sharply, huge cost pressures led to the slowdown in the expansion of 100 stores, and even bring a new round of closing shop tide. At the same time, since 1990s, the network retail industry has experienced four stages: embryonic stage, development period, stable stage and mature stage (Juanjuan Wang, 2015). The report shows that the scale of China's online transactions reached from 800 billion yuan in 2011 to 5 trillion and 200 billion yuan in 2016, but the growth rate has declined year by year. In 2016, China's online shopping market ranked first in the world, and the total volume of trade accounted for more than $10 \%$ of the total retail sales of consumer goods. It is easy to see that the traditional retail industry in the precarious edge of survival struggle, network retail seems to have touched the development of "ceiling", while the electricity supplier to strive for traffic and consumption, in fact, under the line. In October 13, 2016, Ma Yun, chairman of the board of directors of the Alibaba group, made clear for the first time the concept of "new retail" in the 2016 Hangzhou cloud conference, which brought new impacts and opportunities to the entire retail industry.

\section{NEW RETAIL FEATURES}

\subsection{The meaning of new retail}

The first "new retail" concept of Ma, read it as "online, offline and logistics must be combined together, in order to produce the real new retail.Offline enterprises must go up the line, the online business must go down the line, online and offline, coupled with modern logistics together, in order to truly create a new retail." At present, the "new retail" has not yet unified standardized standards concept. Shumei Zhao believes that the so-called "new retail", is the application of the advanced theory and technology of the Internet, improvement and innovation of the traditional retail, with new ideas and thinking as a guide, will sell goods and services to all the activities of the final consumer (Shumei Zhao, Xiaohong Xu, 2017).

\subsection{New retail features}

Different from traditional retail, the new retail is characterized by enhancing the shopping experience of consumers, so that consumers can always buy the goods they need in the shortest possible time. It can be divided into the following three aspects: first, the reconstruction of business factors, from "freight yard man" to "man goods yard", including reengineering the production process, reconstructing the relationship between the business and consumers, and reconstructing the consumption experience. Two is to break the line, online and offline contradictions barriers, increase online and offline integration, to achieve integration of online and offline. Three is to vigorously develop and apply the wisdom of logistics, improve logistics efficiency, reduce logistics costs, enhance consumer shopping experience.

\section{STATUS QUO OF URBAN LOGISTICS DISTRIBUTION}

\subsection{Problems existing in urban logistics distribution}

\subsubsection{The infrastructure of urban logistics and distribution is not perfect}

City logistics facilities by the end of receiving platform, temporary center and distribution center. Business district, office buildings, residential areas and universities and other densely populated, send and receive materials in large quantities, the demand of logistics distribution service is urgent, but the city planning is not perfect, the logistics facilities of commercial district and other populated areas seriously insufficient. The urban expansion brought the dismantling of the existing logistics facilities, the temporary center and the central distribution center have been relocated again and again, and the distribution distance has been increasing. The terminal receiving platform near the traffic congestion and the shortage of parking spaces, delivery vehicles can not at the appointed 
place, bypass and waiting for parking seriously affects the efficiency of distribution.

\subsubsection{The low intensive degree of urban logistics distribution}

At present, there are three main modes of urban logistics distribution: self-built and self-operated, other built and self-operated, and third party logistics. Among them, the self-employed is mainly large chain enterprises, the existence of higher threshold, greater demand for funds, distribution centers difficult location, higher level of management, service upgrades are restricted and other issues; Other built and self-operated are mainly large and medium-sized chain enterprises leased the existing logistics property, self-operate the city logistics distribution, due to the uneven distribution of their own business caused the waste of resources, at the same time, due to the limitation of logistics property, multiplicity investment and management, often running effect is not ideal; Small and medium enterprises mainly choice the third party logistics, because of the limited resources and business, outsourced city logistics distribution to the third party, settled by the single order or on a monthly basis, because the problem of the third party logistics enterprise management and interests, usually in the distribution efficiency and service experience will be greatly reduced. The whole urban logistics distribution industry is difficult to form a big amalgamation, act of their own free will, uneven hunger and fullness, seriously restricting the development of the industry.

\subsubsection{Shortage of government supervision and guidance}

In recent years, because of traffic congestion and environmental pressure, and lack of relevant administrative laws and regulations, governments at all levels to limit truck policy more and more strict, the limit line area is more and more big, the limit line increasingly long time, and the management of electric bicycle as the next direction. For the urban logistics distribution industry, all kinds of policies seriously limit the development of the industry, but the materials of the city logistics distribution is the daily needs of the residents. How to take effective measures to achieve the goal of controlling congestion and environmental protection, while ensuring the daily supply of residents, is the government at all levels should be put on the agenda as soon as possible.

\subsection{Current domestic research results}

In recent years, based on the application of cloud platform and big data analysis, the domestic research results about urban logistics distribution are almost a hundred times to abroad, and most of them take the urban logistics distribution efficiency and the distribution cost into consideration. The following mainly introduces the models and algorithms proposed by scholars at home. In the aspect of distribution efficiency, Junsheng He takes single vehicle multi task and multi vehicle multi task as the research object, establishes the route optimization model and uses heuristic algorithm to solve it, and then gets a more systematic optimized distribution scheme (Junsheng He, 2013). Jian Xu designed the city logistics distribution system of logistics based on the equilibrium, to achieve the distribution system high-end and integrative, constructed three layer structure of the high-end city logistics distribution system which comprised by the planning control layer, supervision layer, business layer layout evaluation(Jian $\mathrm{Xu}$, Hang Liu, Fujuan Yu, 2016). In the aspect of distribution costs, the two stage mathematical model of open dynamic vehicle routing problem of multi vehicle demand and the actual transportation planning problem of Jingling Zhang is fit, including the pre optimization stage and real-time dynamic optimization stage, pre optimization model can solve the known demand distribution planning problem; for the new customer demand, dynamic optimization model can be realized real time optimization(Jingling Zhang, Yanwei Zhao, Haiyan Wang, 2010). In consideration of the efficiency and cost of distribution, Xianlong Ge established a model of inter regional joint distribution of urban logistics, and obtained the optimal distribution plan for traffic restrictions(Xian long Ge, Jue Huang, Baichuan Tan, 2017).

\section{SUGGESTIONS ON THE DEVELOPMENT OF URBAN LOGISTICS DISTRIBUTION UNDER NEW RETAIL}

\subsection{Build collaborative distribution mainly, outsourcing distribution supplemented, self-built and self-operated coexistence model}

Recommended the establishment of city logistics distribution industry association, strengthen the communication between enterprise integration, mergers and acquisitions of small enterprises, to achieve the scale of operation, commit to building the cooperative distribution mode, make full use of social resources, reduce and depress duplication of investment, improve the efficiency of distribution, indirect reduce the congestion of the city. It can be supplemented by outsourcing distribution, so that outsourcing distribution can be integrated into collaborative delivery, and finally form large synergy. Do not exclude self built and self operated, which can be study together and develop together.

\subsection{Build logistics cloud platform and large database to improve the level of urban logistics and distribution informatization}

Set up a cloud integration cloud platform, the city logistics distribution enterprise cloud access platform, through big data analysis, intelligent location the terminal receiving platform, temporary center and distribution center, automatic matching distribution route. Logistics vehicles realize global positioning system (GPS) full access, requiring drivers to strictly follow the automatic matching delivery route. The development of mobile phone client application (APP), real-time monitoring to the truck running position, and be expected to the time of reach the next distribution place, and the time of arrive at the customer site, perfect customer shopping experience of new retail integration.

The application of radio frequency identification 
technology, global positioning system, laser scanner and infrared sensors and other equipment and information technology, build the Internet and goods into Internet of things, realizing data information exchange, to achieve accurate delivery, one-time distribution, reduce the ratio of bad mistakes. In order to meet the requirements of the new retail environment, in the completion of delivery, taking into account the return, replacement goods demand, to achieve a delivery, once disposes, to avoid duplication using of resources, resulting in waste.

\subsection{Developing automation equipment, exploring intelligent logistics and green logistics}

Because the land and labor logistics industry basic factors of production costs rose sharply, the new retail massive orders "fast", "quasi" and "real time" and "transparent", and the flexible demand of modern manufacturing, the development of urban logistics distribution automation equipment has been imminent. To realize cooperation with online and offline of the new retail needs to be done during the peak period of distribution logistics distribution nearest, and the same price paid of online and offline hereinafter; Online orders, the store near the delivery, or offline orders, online delivery. The pain point of the electricity supplier industry is difficult of capacity dispatching transport by the demand of tide, the pain point of the fresh industry is the timeliness of the control, the pain point of the supermaket industry is within the specified time to complete multi-point distribution. The urban logistics distribution period overlaps with the busy traffic time, and the distribution efficiency is low.

Explore the development of intelligent logistics, green logistics, solve the pain points of enterprises and logistics industry difficulties, the use of new energy and low fuel consumption distribution vehicles. Innovative high-tech applications, equipment, automated warehousing system, the development of UAV distribution, unmanned delivery vehicles. Create multichannel distribution mode, such as urban light rail distribution, taxi distribution, etc...

\subsection{The government's active guidance industrial upgrading}

The government should formulate and improve relevant administrative laws and regulations to guide, encourage, and even reward urban logistics distribution enterprises to carry out the upgrading of intelligent logistics and green logistics industry. Considering the logistics infrastructure construction of city planning and construction, opening the path to the logistics, such as logistics private channel, logistics special parking lot, special parking space for logistics and so on, then transferred the personnel to the ground or the air, human resources and logistics achieve separation, reduce cross congestion.

\section{CONCLUSIONS}

New retail has arrived, the traditional urban logistics and distribution has been unable to adapt, and seriously restricting the development of new retail. In order to realize the new retail, city logistics distribution mode need innovation, use big data and cloud technology, enhance the level of information, at the same time, develop automation equipment, explore the wisdom of logistics and green logistics, and actively guide by the government, to reduce the cost of urban logistics distribution, to enhance the efficiency of the city logistics distribution, to jointly promote the new retail lively.

\section{REFERENCES}

[1] Juanjuan, Wang, 2015. To explore the the belt and road strategic area electronic commerce new normal mode, Chinese circulation economy. 2015(4), pp.46-54.

[2] Shumei, Xu, Xiaohong, Xu, 2017. The meaning, model and development path of "new retail", Chinese circulation economy 2017(5), pp.12-20.

[3] Junsheng, He, 2013. Research on optimization of delivery route model in express industry. Chongqing: Chongqing Jiaotong University (Master degree thesis).

[4] Jian, Xu, Hang Liu, Fujuan, Yu, 2016. Design of urban logistics distribution system based on logistics equilibrium. Logistics technology. 2016(2), pp.27-30.

[5] Jingling, Zhang, Yanwei, Zhao, Haiyan, Wang, 2010. Modeling and optimization of vehicle routing problem with multi vehicle dynamic demand. Computer integrated manufacturing system. 2010(3), pp.543-550.

[6] Xianlong, Ge, Jue, Huang, Baichuan, Tan, 2017. Model and algorithm of inter-regional joint distribution of urban logistics. Control and decision. 2017(5), pp.789-796. 